\title{
Kinetik der Bromat-Nitritreaktion
}

\author{
(Nachtrag) \\ von
}

\section{Albin Kurtenacker.}

Aus dem Laboratorium für anorganische, physikalische und analytische Chemie der Deutschen Technischen Hochschule in Brünn.

(Vorgelegt in der Sitzung am 29. April 1915.)

Eine im Vorjahre ${ }^{1}$ mitgeteilte kinetische Untersuchung der Bromat-Nitritreaktion ergab die auffallende Tatsache, daß die Geschwindigkeit dieser Reaktion von der Nitritkonzentration vollständig unabhängig ist, vorausgesetzt, daß das Nitrit im Überschuß gegenüber dem Bromat angewendet wird. Aus diesem Verhalten wurde gefolgert, daß nicht die salpetrige Säure selbst durch Bromsäure oxydiert wird, sondern deren in wässeriger Lösung entstehendes Zersetzungsprodukt, das Stickoxyd.

Im folgenden sollen einige Versuche mitgeteilt werden, die weitere Belege für die Richtigkeit dieser Auffassung des Reaktionsvorganges bilden.

Als Richtschnur für die Ausführung dieser Versuche diente die Überlegung, daß die Beteiligung des Stickoxyds

1 Monatshefte für Chemie, 35, 925 (1914). - Berichtigung: In den Tabellen dieser Arbeit wurden die Essigsäurekonzentrationen irrtümlich um $4 \% \mathrm{zu}$ hoch angegeben. In den Tabellen 1 bis 11 soll es daher heißen statt $\mathrm{CH}_{3} \mathrm{COOH} 2: 1.92$, in Tabelle 14 statt $0.5: 0.48$, in Tabelle 15 statt 1 : $0 \cdot 96$, in Tabelle 16 statt $3: 2 \cdot 88$, in Tabelle 17 statt $4: 3 \cdot 84$. Die übrigen Zahlen und die Ergebnisse der Arbeit werden durch diese Änderung natürlich nicht beeinflußt. 
an der Reaktion eine charakteristische Abhängigkeit der Reaktionsgeschwindigkeit von Druck und Temperatur bedingen müsse.

Am einfachsten liegen die Verhältnisse, wenn man die Wirkung des Druckes untersucht. Reaktionen in Lösungen von festen und flüssigen Substanzen sind bekanntlich vom Druck vollständig unabhängig, wenn man nicht Pressungen von mehreren hundert Atmosphären anwendet. Ist hingegen Stickoxyd, also ein Gas, an der Reaktion beteiligt, so muß die Reaktionsgeschwindigkeit sich ganz erheblich mit dem Druck ändern, weil schon eine geringe Variation des Druckes eine starke Änderung der Stickoxydkonzentration in der Lösung bewirkt. Umgekehrt wird man aus einer etwa beobachteten starken Beeinflussung der Reaktionsgeschwindigkeit durch den Druck mit Sicherheit schließen können, daß die Reduktion der Bromsäure durch Stickoxyd bewirkt wird.

Aus der Änderung der Reaktionsgeschwindigkeit mit der Temperatur ist die Beteiligung des Stickoxyds nicht so klar ersichtlich, weil die Temperatur die Reaktionsgeschwindigkeit in zweifacher Hinsicht beeinflussen kann. Einmal wird jedenfalls, wie bei allen Reaktionen, eine bedeutende Reaktionsbeschleunigung bewirkt we:den, außerdem kann aber, falls das Stickoxyd eine Reaktionskomponente bildet, gleichzeitig eine Verzögerung eintreten, die der Abnahme der Löslichkeit des Stickoxyds mit steigender Temperatur entspricht. Da sich die beiden Wirkungen der Temperatur überdecken, muß die reine Temperaturbeschleunigung rechnerisch ermittelt werden, damit die eventuell durch das Stickoxyd bewirkte Verzögerung zum Ausdruck kommt. Zur Berechnung der Temperaturbeschleunigung wurde die bekannte Arrhenius'sche Gleichung $\frac{d l K}{d T}=\frac{A}{T^{2}} 1$ benützt. $\mathrm{Ob}$ diese Gleichung für die in Rede stehende Reaktion streng gilt, läßt sich allerdings nicht feststellen; es ist aber anzunehmen, daß die Abweichung von der Gleichung nur klein sein wird, da die Temperaturbeschleunigung der meisten bisher untersuchten Reaktionen

1 Zeitschr. phys. Chem., 4, 226. 
der Gleichung vollkommen entspricht. ${ }^{1}$ Eine starke, im Sinne der Löslichkeitsverminderung des Stickoxyds liegende Abweichung wird demgemäß auf eine Wirksamkeit der Stickoxydkonzentration schließen lassen und eine weitere Bestätigung für die Richtigkeit der oben über den Reaktionsverlauf gemachten Annahme bilden.

Einfluß des Druckes.

$\mathrm{Da}$ das Arbeiten unter höherem als Atmosphärendruck eine komplizierte Apparatur erfordert, wurden die Versuche bei vermindertem Druck ausgeführt. In einem dickwandigen Glaskolben wurde Kalumnitritlösung mit Essigsäure und so viel Wasser gemischt, daß wie bei den früheren Versuchen ein Reaktionsvolumen von $100 \mathrm{~cm}^{3}$ erhalten wurde. Die Kaliumbromatlösung wurde in einem offenen Fläschchen gesondert in den Kolben eingebracht. Hierauf wurde der Kolben verschlossen, mit der Wasserstrahlpumpe evakuiert und zur Erzielung des Temperaturausgleiches eine Viertelstunde im Thermostaten stehen gelassen. Nach dieser Zeit wurde durch Umschütteln mit der Kaliumbromatlösung gemischt und damit die Reaktion eingeleitet. Nach der gewünschten Zeit wurde die Reaktion dadurch zum Stillstand gebracht, daß durch einen Hahntrichter soviel Natronlauge einfließen gelassen wurde, als zur Absättigung von etwa $80 \%$ der angewendeten Essigsäure erforderlich war. Die Bestimmung des unzersetzt gebliebenen Bromats geschah wie bei den früher mitgeteilten Versuchen auf jodometrischem. Wege nach Oxydation der salpetrigen Säure durch Bromwasser und Entfernen des Bromüberschusses durch Thiosulfat.

Die in den Tabellen 1 und 2 zusammengestellten Versuche wurden bei einer Temperatur von $20^{\circ}$ mit folgenden Anfangskonzentrationen an Reagenzien ausgeführt:

$$
\mathrm{KBrO}_{3}: 0.005 ; \mathrm{KNO}_{2}: 0.02 ; \mathrm{CH}_{3} \mathrm{COOH}: 2
$$

(die Konzentrationen sind in denselben Einheiten angegeben wie in der früheren Arbeit).

1 Siehe u. a. v. Halban, Zeitschr. phys. Chem., 67, 172. 
Die Versuche in Tabelle 1 wurden bei Atmosphärendruck $(730 \mathrm{~mm})$ durchgeführt, die Versuche in Tabelle 2 bei einem Druck von 38 bis $40 \mathrm{~mm} \mathrm{Hg}$.

Tabelle 1.

Temperatur: $20 \cdot 0^{\circ}$.

Druck: $730 \mathrm{~mm} \mathrm{Hg}$.

\begin{tabular}{|r|r|r|}
\hline$t$ & $A-x$ & \multicolumn{1}{|c|}{$K$} \\
\hline \hline 0 & $30 \cdot 20$ & \multicolumn{1}{c|}{-} \\
2 & $23 \cdot 13$ & $0 \cdot 0579$ \\
4 & $17 \cdot 85$ & 571 \\
6 & $13 \cdot 57$ & 579 \\
8 & $10 \cdot 46$ & 576 \\
10 & $8 \cdot 10$ & 572 \\
13 & $5 \cdot 43$ & 573 \\
16 & $3 \cdot 70$ & 570 \\
\hline & Mittel... & $0 \cdot 0574$ \\
& &
\end{tabular}

Tabelle 2.

Temperatur: $20 \cdot 0^{\circ}$.

Druck: 38 bis $40 \mathrm{~mm} \mathrm{Hg}$.

\begin{tabular}{|r|r|r|}
\hline \multicolumn{1}{|c|}{$t$} & $A-x$ & \multicolumn{1}{c|}{$K$} \\
\hline \hline 0 & $30 \cdot 30$ & \multicolumn{1}{c|}{-} \\
2 & $24 \cdot 23$ & 0.0486 \\
4 & $19 \cdot 25$ & 493 \\
6 & $15 \cdot 33$ & 493 \\
8 & $12 \cdot 12$ & 497 \\
10 & $9 \cdot 86$ & 488 \\
13 & $6 \cdot 91$ & 495 \\
16 & $5 \cdot 09$ & 484 \\
& Mittel... & 0.0491 \\
& & \\
& &
\end{tabular}

Beide Versuchsreihen ergaben, wie ersichtlich, eine sehr befriedigende Konstanz der Geschwindigkeitskoeffizienten erster Ordnung, infolge Vermeidung kleiner Titrationsfehler sogar eine bessere als die in der ersten Arbeit angegebenen Versuche.

Der Einfluß des Druckes auf die Reaktionsgeschwindig. keit geht aus dem Vergleich der Mittelwerte von $K$ hervor. Der angewandten Druckdifferenz entspricht die ganz bedeutende Verlangsamung der Reaktion um etwa 15\% ihrer ursprünglichen Geschwindigkeit. Andere Versuche, die mit abweichenden Reagenzienkonzentrationen ausgeführt wurden, führten zu ähnlichen Resultaten, weshalb sich ihre Mitteilung hier erübrigt.

Die Abhängigkeit der Reaktionsgeschwindigkeit vom Druck beweist nach obigem, daf die Bromsäure durch Stickoxyd und nicht durch salpetrige Säure reduziert wird. Die in der ersten Mitteilung gemachte Annahme, daß die Löslichkeit des Stickoxyds allein geschwindigkeitsbestimmend sei, kann 
freilich nicht voll aufrecht erhalten werden, weil dem angewandten Druckunterschied in diesem Fall eine bedeutend größere Verzögerung der Reaktion hätte entsprechen müssen. Es ist deshalb anzunehmen, daß noch ein anderer Faktor eine Rolle spielt, wahrscheinlich die im Vakuum erhöhte Zersetzungsgeschwindigkeit der salpetrigen Säure und die hiedurch bedingte raschere Nachlieferung des Stickoxyds.

\section{Einfluf der Temperatur.}

Die Versuche wurden genau so ausgeführt, wie in der ersten Arbeit beschrieben wurde. Die einzelnen Stoffe kamen bei allen Versuchen in den p. 453 angegebenen Konzentrationen zur Anwendung. Die Ergebnisse sind in den Tabellen 1, 3, 4 und 5 zusammengestellt.

Tabelle 3 .

Temperatur: $30 \cdot 0^{\circ}$.

\begin{tabular}{|c|c|c|}
\hline$t$ & $A-x$ & $K$ \\
\hline 0 & $30 \cdot 20$ & - \\
\hline 1 & $22 \cdot 45$ & 0.129 \\
\hline 2 & $16 \cdot 65$ & 129 \\
\hline 3 & $12 \cdot 40$ & 129 \\
\hline 4 & $9 \cdot 35$ & 127 \\
\hline 6 & $5 \cdot 20$ & 127 \\
\hline \multirow[t]{2}{*}{8} & $2 \cdot 90$ & 127 \\
\hline & & 0.128 \\
\hline
\end{tabular}

Tabelle 4.

Temperatur: $40 \cdot 0^{\circ}$.

\begin{tabular}{|c|r|r|}
\hline$t$ & $A-x$ & \multicolumn{1}{c|}{$K$} \\
\hline \hline 0 & 30.20 & \multicolumn{1}{c|}{-} \\
1 & 16.65 & 0.259 \\
2 & 9.00 & 263 \\
3 & 4.93 & 262 \\
4 & 2.87 & 256 \\
5 & 1.57 & 257 \\
6 & 1.00 & 247 \\
& Mittel... & 0.257 \\
\hline
\end{tabular}

Tabelle 5.

Temperatur: $50 \cdot 0^{\circ}$.

\begin{tabular}{|c|r|r|}
\hline$t$ & \multicolumn{1}{|c|}{$A-x$} & \multicolumn{1}{|c|}{$K$} \\
\hline \hline 0 & $30 \cdot 20$ & \multicolumn{1}{c|}{-} \\
1 & $10 \cdot 03$ & $0 \cdot 479$ \\
2 & $3 \cdot 30$ & 481 \\
3 & $1 \cdot 32$ & 453 \\
4 & $0 \cdot 40$ & 470 \\
& Mittel... & $0 \cdot 471$ \\
\hline
\end{tabular}

Chemie-Heft Nr. 6 . 
Um zu prüfen, ob die erwartete Abweichung von der Arrhenius'schen Gleichung stattfindet, wurde die Gleichung in der integrierten Form

$$
\log \frac{K_{1}}{K_{2}}=A \frac{T_{1}-T_{2}}{T_{1} T_{2}}
$$

angewendet. Darin sind mit $K_{1}$ und $K_{2}$ die Geschwindigkeitskonstanten zweier Versuche bezeichnet, die bei den absoluten Temperaturen $T_{1}$ und $T_{2}$ ausgeführt wurden. $A$ muß, wenn die Gleichung befriedigt sein soll, eine Konstante sein; nimmt die Reaktionsgeschwindigkeit hingegen, wie vermutet, mit der Temperatur langsamer $z u$, als der Gleichung entspricht, so müssen die Werte von $A$ einen abnehmenden Gang zeigen.

In der folgenden Tabelle sind die zu den absoluten Temperaturen $T$ gehörigen Mittelwerte der Koeffizienten $K$ eingetragen. In der letzten Rubrik ist $A$ aus je zwei aufeinanderfolgenden Versuchen berechnet.

Tabelle 6.

\begin{tabular}{|c|c|c|}
\hline$T$ & $K$ & $A .10^{-1}$ \\
\hline \hline 293 & 0.0574 & - \\
303 & 0.128 & 309 \\
313 & 0.257 & 287 \\
323 & 0.471 & 266 \\
\hline
\end{tabular}

Das Verhalten der Reaktion bei Temperaturerhöhung entspricht demnach ebenfalls dem angenommenen Reaktionsverlauf. 